\title{
Virtual Dermatology and the COVID-19 Pandemic in a Resource-Limited Country Such as Nepal
}

\author{
Krishna Jha, MD; Anil Kumar Jha, MD \\ Dermatology Institute Skin Health and Referral Center, Maharajgunj, Kathmandu, Bagmati Province, Nepal
}

\section{Corresponding Author:}

Anil Kumar Jha, MD

Dermatology Institute Skin Health and Referral Center

Maharajgunj

Maharajgunj Road

Kathmandu, Bagmati Province, 44600

Nepal

Phone: 97714721500

Email: dranilkjha@hotmail.com

\section{Abstract}

Background: The COVID-19 pandemic has caused nationwide lockdown, which led to the disruption of health services. Despite being a rising health care modality in Nepal, virtual dermatology services became an effective tool to provide dermatologic care through web-based consultations throughout the country. Therefore, we assessed the implementation of teledermatology services at our center to provide uninterrupted health services across the country during the pandemic.

Objective: This study aimed to evaluate the clinicodemographic profile of patients using teledermatology services and patient acceptance of this service.

Methods: A retrospective, single-center, observational study was carried out. Clinicodemographic data from the patients using teledermatology services were obtained and analyzed. A set of questionnaires regarding patients' acceptance of teledermatology services were administered to the patients through a survey via telephone calls, and the obtained data were interpreted.

Results: A total of 122 teleconsultations were carried out within the country. The mean age of patients was 33.48 (SD 17.89) years. Of these 122 patients, $79(64.8 \%)$ were from outside and $43(35.2 \%)$ were from inside the city where the institute is located. The average distance from the institute to the patients' residence was approximately 144.84 (SD 157.20) km, and the mean travel time was approximately 385.31 (SD 889.52) minutes. In total, 89 patients could be contacted, of whom 81 ( $91 \%$ ) found the service easy to use, $75(84.3 \%)$ were able to express their problems in a manner similar to that during direct visits, $49(55.05 \%)$ thought that the teleconsultation was the same as an in-person visit, $80(89.9 \%)$ were satisfied, and $85(95.5 \%)$ agreed to use teledermatology services in the future. Superficial fungal infection was the most common diagnosis $(24.6 \%)$. Newly registered patients were more satisfied than follow-up patients $(96.36 \%$ vs $79.41 \%$, respectively; $P=.01)$.

Conclusions: This study highlights the importance of virtual dermatology services to deliver dermatologic care during the pandemic in Nepal. In the future, this program has a promising role in providing health care services to meet the medical needs of patients.

Conflicts of Interest: None declared.

(iproc 2022;8(1):e36892) doi: $10.2196 / 36892$

\section{KEYWORDS}

virtual dermatology; teledermatology; COVID-19; pandemic; resource-poor setting; Nepal 
Edited by T Derrick; this is a non-peer-reviewed article. Submitted 28.01.22; accepted 28.01.22; published 08.02.22.

Please cite as:

Jha K, Jha AK

Virtual Dermatology and the COVID-19 Pandemic in a Resource-Limited Country Such as Nepal iproc 2022;8(1):e36892

URL: https://www.iproc.org/2022/1/e36892

doi: $\underline{10.2196 / 36892}$

PMID:

(OKrishna Jha, Anil Kumar Jha. Originally published in Iproceedings (https://www.iproc.org), 08.02.2022. This is an open-access article distributed under the terms of the Creative Commons Attribution License (https://creativecommons.org/licenses/by/4.0/), which permits unrestricted use, distribution, and reproduction in any medium, provided the original work, first published in Iproceedings, is properly cited. The complete bibliographic information, a link to the original publication on https://www.iproc.org/, as well as this copyright and license information must be included. 